\title{
On properties of solutions for Fokker-Planck-Kolmogorov equations
}

\author{
Medynsky I. P. \\ Lviv Polytechnic National University, \\ 12 S. Bandera Str., 79013, Lviv, Ukraine
}

(Received 17 March 2020; Revised 20 April 2020; Accepted 22 April 2020)

\begin{abstract}
In the paper, we illuminate the connection between diffusion processes and partial differential equations of parabolic type. The emphasis is on degenerate parabolic equations with real-valued coefficients. These equations are the generalization of the classical Kolmogorov equation of diffusion with inertia, which may be treated as Fokker-Planck-Kolmogorov equations for the corresponding degenerate diffusion processes. A fundamental solution of the Cauchy problem for Fokker-Planck-Kolmogorov equation determines the transition probabilities to the corresponding diffusion process. The conditions on the coefficients under which there exists the classical fundamental solution are formulated. The basic properties of fundamental solutions are proved. The application of the fundamental solution to the investigation of correct solvability for the Cauchy problem is presented.
\end{abstract}

Keywords: diffusion process, transition probability to a process, Fokker-PlanckKolmogorov equation, degenerate parabolic equation, fundamental solution, Cauchy problem.

2010 MSC: $35 \mathrm{~K} 15,35 \mathrm{~K} 65,60 \mathrm{~J} 60$

DOI: $10.23939 / \mathrm{mmc} 2020.01 .158$

\section{Introduction}

In this paper, we consider equations from the class $\mathbf{E}_{22}$ (definition of this class is given in monograph [1]) with real-valued coefficients. These equations often appear in the investigations of various physical phenomena in the so-called diffusion approximation. In many important cases (for example, in the study of the Brownian motion) these mathematical models describe natural phenomena adequately and in a relatively simple way. Usually such equations, used systematically from the beginning of the last century, are called the Fokker-Planck equations. These equations often determine the continuous time evolution of a Markov stochastic process. Studying models of the Brownian motion, Kolmogorov [2] selected the class of continuous Markov processes, which later get the name of diffusion processes. In the mentioned work, Kolmogorov showed that diffusion processes are closely connected with the second-order partial differential equations of the parabolic type. Namely, if the function $P(s, x, t, \Gamma)$ determines the transition density to the diffusion process with the drift vector $a$ and diffusion matrix $b$, then under such conditions, the function

$$
u(s, x):=\int_{\mathbb{R}^{m}} \varphi(y) P(s, x, t, d y)
$$

is a solution in layer $\left\{(s, x) \mid s \in[0, t), x \in \mathbb{R}^{m}\right\}$ for the equation

$$
\partial_{s} u+\frac{1}{2} \sum_{j, k=1}^{m} b_{j k}(s, x) \partial_{x_{j}} \partial_{x_{k}} u+\sum_{j=1}^{m} a_{j}(s, x) \partial_{x_{j}} u=0
$$

with the initial condition

$$
\lim _{s \rightarrow t+} u(s, x)=\varphi(x) .
$$

Here $b_{j k}(s)$ are the elements of the matrix $b, a_{j}$ are coordinates of a vector $a$, and $x_{j}$ are coordinates of a vector $x$. From the diffusion view on the phenomenon, the drift vector $a$ is a macroscopic velocity of liquid, and the diffusion matrix $b$ characterizes random movement of a participle, which are the result of collisions with molecules of liquid, which are in a thermomotion. 
Besides the inverse equation (2), the so-called direct equation is obtained in this work, too. Both equations are the second-order partial differential equations of parabolic type.

Let us pay attention to the fact that direct equations for some special cases have been got by the physicists Fokker and Planck a bit earlier, who studied phenomenon of diffusion. That is why it is also called the Fokker-Planck equation.

In the classical theory of the Brownian motion developed by Einstein and Smoluchowski $[3,4]$ the inertia of a Brownian particle is neglected, that is the mass of a particle is actually assumed to equal 0. Therefore, a Brownian particle in the Einstein-Smoluchowski theory cannot have a finite velocity. Brownian motion of a physical system in the Einstein-Smoluchowski approximation is a continuous Markov process on the coordinate space (the Wiener process, for the case of a free particle).

The fact of non-differentiability of Brownian paths in the Einstein-Smoluchowski theory is closely connected with an idealization made in this theory (the neglect of inertia) making it invalid on small time intervals. For the simplest case of the Brownian motion of a free particle, a theory taking inertia into account was developed as early as in 1930 by Uhlenbeck and Ornstein [5]. In this more precise theory, paths are already differentiable (but do not have the second derivative, so that now the acceleration becomes infinite).

In fact, the same generalization is contained in the paper [6] by A. N. Kolmogorov. He considered the general case of the Brownian motion for an arbitrary physical system with $n$ degrees of freedom. According to Kolmogorov, inertia is taken into account if a state of the system is described by values of $n$ coordinates $q_{1}, \ldots, q_{n}$ and $n$ their time derivatives (velocities) $\dot{q}_{1}, \ldots, \dot{q}_{n}$. Here the model of the Brownian motion is a continuous Markov process in the $2 n$-dimensional phase space of coordinates and velocities.

In the paper [6] is assumed that, whenever we know the values of $q:=\left(q_{1}, \ldots, q_{n}\right)$ and $\dot{q}:=$ $\left(\dot{q}_{1}, \ldots, \dot{q}_{n}\right)$ at an instant of time $t$, we can find the probability density $G\left(t, q, \dot{q} ; t^{\prime}, q^{\prime}, \dot{q}^{\prime}\right)$ of possible values $q$ and $\dot{q}$ of the coordinates and their time derivatives at an arbitrary instant $t^{\prime}>t$. It is assumed that $G$ does not depend on the behavior of the system before the instant $t$ (there is no aftereffect, the process is of the Markov type). It is proved that the function $G$ is a fundamental solution of the Cauchy problem (FSCP) for the Fokker-Planck differential equation. Thus, it is natural to call them the Fokker-Planck-Kolmogorov equations for the corresponding degenerate diffusion processes. Such equations belong to a class ultraparabolic or elliptic-parabolic equations.

The development of the theory for ultraparabolic equations of the Kolmogorov type, subsequent investigations aimed at finding as a more weak conditions as possible for the existence of a FSCP, obtaining its precise estimates, considering equations with a more complicated structure. Investigation of FSCP and correct solvability of the Cauchy problem for degenerate parabolic equations of the Kolmogorov type under different assumption for coefficients of equations worked out M. Weber, A. M. Il'in, I. M. Sonin, Ya. I. Shatyro, L. P. Kuptsov, S. D. Eidelman, A. P. Malitska, L. M. Tychinska. S. D. Ivasyshen, L. N. Androsova and V. S. Dron' (see monograph [1]).

Well known [7-9], that nondegenerate diffusion processes with sufficiently regular characteristics possess smooth transition densities. In general, a degeneration of the diffusion matrix leads to the nonexistence of a density of the transition probability. However there exist some classes of degenerate processes with smooth transition densities. The processes of the Brownian motion with inertia considered by A. N. Kolmogorov, A. M. Il'in, R. Z. Khasminsky and others are among such processes. In the paper [10] I. M. Sonin studied a natural generalization of the process of diffusion with inertia. For the class of processes considered the transition densities are constructed as FSCP for the equations

$$
\begin{aligned}
\partial_{t} u=\left(\frac{1}{2} \sum_{j, l=1}^{n} a_{j l}(t, x, y, z) \partial_{y_{j}} \partial_{y_{l}}\right. & +\sum_{j=1}^{n} a_{j}(t, x, y, z) \partial_{y_{j}} \\
& \left.+\sum_{j=1}^{n} b_{j}(t, x, y, z) \partial_{x_{j}}+\sum_{j=1}^{n} c_{j}(t, x, y, z) \partial_{z_{j}}\right) u, \quad\{x, y, z\} \subset \mathbb{R}^{n},
\end{aligned}
$$

where $b_{j}(t, x, y, z)$ behave "approximately" like $y_{j}$, and $c_{j}(t, x, y, z)$ as $x_{j}$. Existing a FSCP for the equation (4) under condition that coefficients of the equation are sufficiently smooth functions is proved. 
Note that, the results of constructing and investigation properties of a FSCP for the equations, which have the form (4) under more weakly conditions for their coefficients is contained in the monograph [1] and the paper [11]. Some newly results about FSCP and correct solvability of the Cauchy problem for the equations type of (4) we will announce in the following sections. Interest in obtaining results for equations from the class $\mathbf{E}_{22}$ is not academic. Some motivations for studying ultraparabolic equations of Kolmogorov type from the stochastic theory of diffusion processes, statistical physics, financial mathematics and especially option theory are given in papers [1,11-19]. Different properties of the probability solutions for linear and nonlinear Fokker-Planck-Kolmogorov equations are presented in $[20]$ and $[21]$.

\section{FSCP for the Fokker-Planck-Kolmogorov equations of degenerate diffusion pro- cesses}

The main object of our study, in this section the FSCP for some degenerate parabolic equations, can be interpreted in a natural way as a transition density of a respectively diffusion process with a value from $n$-dimension phase space $\mathbb{R}^{n}$ of points $x$ with three different groups of phase coordinates $x_{s}:=\left(x_{s 1}, \ldots, x_{s n_{s}}\right) \in \mathbb{R}^{n_{s}}, s \in\{1,2,3\}, n=n_{1}+n_{2}+n_{3}, 1 \leqslant n_{3} \leqslant n_{2} \leqslant n_{1}$.

We consider the equation with real-valued coefficients

$$
\left(S-\sum_{j, l=1}^{n_{1}} a_{j l}(t, x) \partial_{x_{1 j}} \partial_{x_{1 l}}-\sum_{j=1}^{n_{1}} a_{j}(t, x) \partial_{x_{1 j}}-a_{0}(t, x)\right) u(t, x)=f(t, x), \quad(t, x) \in \Pi_{(0, T]},
$$

and the corresponding adjoint equation

$$
\begin{aligned}
& S^{*} v(\tau, \xi)-\sum_{j, l=1}^{n_{1}} \partial_{\xi_{1 j}} \partial_{\xi_{1 l}}\left(a_{j l}(\tau, \xi) v(\tau, \xi)\right)+\sum_{j=1}^{n_{1}} \partial_{\xi_{1 j}}\left(a_{j}(\tau, \xi) v(\tau, \xi)\right) \\
& -a_{0}(\tau, \xi) v(\tau, \xi)=g(\tau, \xi), \quad(\tau, \xi) \in \Pi_{[0, T)} .
\end{aligned}
$$

where $\Pi_{H}:=\left\{(t, x) \mid t \in H, x \in \mathbb{R}^{n}\right\}$, if $H \subset \mathbb{R} ; T$ is given positive number; $S$ is differential expression, which determine by formula

$$
S:=\partial_{t}-\sum_{j=1}^{n_{2}} x_{1 j} \partial_{x_{2 j}}-\sum_{j=1}^{n_{3}} x_{2 j} \partial_{x_{3 j}}
$$

or Li derivative respect to vector field define this expression, and $S^{*}$ is adjoint expression to $S$.

We shall use the Green-Ostrogradsky formula which, for the present situation, takes the form

$$
\begin{array}{rl}
\int_{t_{1}}^{t_{2}} & d \theta \int_{B_{R}}\left(v L u-u L^{*} v\right)(\theta, y) d y=\left.\int_{B_{R}}(v u)(\theta, y)\right|_{\theta=t_{1}} ^{t_{2}} d y \\
& -\int_{t_{1}}^{t_{2}} d \theta \int_{\Gamma_{R}}\left(\sum_{j=1}^{n_{2}} y_{1 j} \mu_{2 j}+\sum_{j=1}^{n_{3}} y_{2 j} \mu_{3 j}\right)(v u)(\theta, y) d S_{y}+\int_{t_{1}}^{t_{2}} d \theta \int_{\Gamma_{R}} \sum_{j=1}^{n_{1}} B^{j}[v, u](\theta, y) \mu_{1 j} d S_{y},
\end{array}
$$

where $t_{1}<t_{2}, B_{R}$ is the ball $\left\{y \in \mathbb{R}^{n}|| y \mid \leqslant R\right\}, \Gamma_{R}$ is its boundary, $\left(\mu_{11}, \ldots, \mu_{1 n_{1}}, \mu_{21}, \ldots, \mu_{2 n_{2}}, \mu_{31}, \ldots\right.$, $\left.\mu_{3 n_{3}}\right)$ is the unit vector of the outer normal to $\Gamma_{R}, L$ and $L^{*}$ are the differential expressions from (5) and (6), $B^{j}[v, u], j \in\left\{1, \ldots, n_{1}\right\}$, are bilinear forms containing derivatives in $y_{1}$ of $v$ and $u$ of the orders not exceeding 1 .

$$
B^{j}[v, u]:=-\sum_{l=1}^{n_{1}}\left(a_{j l} \partial_{y_{1 l}} u v-u \partial_{y_{1 l}}\left(a_{j l} v\right)\right)+a_{j} u v, \quad j \in\left\{1, \ldots, n_{1}\right\} .
$$


Passing to the limit, as $R \rightarrow \infty$, we obtain, for appropriate functions $u$ and $v$, that

$$
\int_{t_{1}}^{t_{2}} d \theta \int_{\mathbb{R}^{n}}\left(v L u-u L^{*} v\right)(\theta, y) d y=\left.\int_{\mathbb{R}^{n}}(v u)(\theta, y)\right|_{\theta=t_{1}} ^{t_{2}} d y
$$

This formula will be used frequently.

Before making an assumptions on the coefficients for the equation (5), we need to introduce the following notations: $\Delta_{x}^{z} f(\cdot, x, \cdot):=f(\cdot, x, \cdot)-f(\cdot, z, \cdot), \Delta_{x_{s}}^{z_{s}} f(\cdot, x, \cdot):=\Delta_{x}^{z^{(s)}} f(\cdot, x, \cdot), s \in \mathbb{N}_{3}, \xi^{(0)}:=\xi$, $\xi^{(1)}:=\left(\xi_{1}, x_{2}, x_{3}\right), \xi^{(2)}:=\left(x_{1}, \xi_{2}, x_{3}\right), \xi^{(3)}:=\left(x_{1}, x_{2}, \xi_{3}\right) ; \xi^{(0)}:=x, z^{(1)}:=\left(z_{1}, x_{2}, x_{3}\right), z^{(2)}:=$ $\left(x_{1}, z_{2}, x_{3}\right), z^{(3)}:=\left(x_{1}, x_{2}, z_{3}\right) ; x^{(1)}:=\left(x_{1}, z_{2}, z_{3}\right), x^{(2)}:=\left(x_{1}, x_{2}, z_{3}\right) ; X(t):=\left(X_{1}(t), X_{2}(t), X_{3}(t)\right)$, $X^{(1)}(t):=\left(\lambda_{1}, X_{2}(t), X_{3}(t)\right), X^{(2)}(t):=\left(\lambda_{1}, \lambda_{2}, X_{3}(t)\right), X_{1}(t):=x_{1}, X_{2}(t):=x_{2}+t \hat{x}_{1}, X_{3}(t):=$ $x_{3}+t x_{2}^{\prime}+2^{-1} t^{2} x_{1}^{\prime}, t \in \mathbb{R}, \hat{x}_{1}:=\left(x_{11}, \ldots, x_{1 n_{2}}\right), x_{1}^{\prime}:=\left(x_{11}, \ldots, x_{1 n_{3}}\right), x_{2}^{\prime}:=\left(x_{21}, \ldots, x_{2 n_{3}}\right), Z^{(s)}(t):=$ $\left.X(t)\right|_{x_{s}=z_{s}}, s \in \mathbb{N}_{3}, Z^{(0)}(t):=X(t)$.

For real-valued coefficients of equation (5) we shall use the following conditions:

1) there exists such a constant $\delta>0$, that for any $(t, x) \in \Pi_{[0, T]}$ and $\sigma_{1} \in \mathbb{R}^{n_{1}}$ the following inequality is valid

$$
\sum_{j, l=1}^{n_{1}} a_{j l}(t, x) \sigma_{1 j} \sigma_{1 l} \geqslant \delta\left|\sigma_{1}\right|^{2} ;
$$

2) the coefficients $a_{j l}, a_{j}$ and $a_{0}$ are bounded and Hölder continuous in following sense:

$$
\begin{gathered}
\exists H_{1}>0 \exists \alpha_{1} \in(0,1) \forall\left\{(t, x),\left(t, z^{(1)}\right)\right\} \subset \Pi_{[0, T]}:\left|\Delta_{x_{1}}^{z_{1}} a(t, x)\right| \leqslant H_{1}\left|x_{1}-z_{1}\right|^{\alpha_{1}}, \\
\exists H_{2}>0 \exists \alpha_{2} \in(1 / 3,2 / 3] \forall\left\{(t, x),\left(t, z^{(2)}\right)\right\} \subset \Pi_{[0, T]} \forall h \in[0, T]: \\
\left|\Delta_{x_{2}}^{z_{2}} a(t, x)\right| \leqslant H_{2}\left(h^{m_{2} \alpha_{2}}+\left|X_{2}(h)-z_{2}\right|^{\alpha_{2}}\right), \\
\exists H_{3}>0 \exists \alpha_{3} \in(3 / 5,2 / 3] \forall\left\{(t, x),\left(t, z^{(3)}\right)\right\} \subset \Pi_{[0, T]} \forall h \in[0, T]: \\
\left|\Delta_{x_{3}}^{z_{3}} a(t, x)\right| \leqslant H_{3}\left(h^{m_{3} \alpha_{3}}+\left|X_{3}(h)-z_{3}\right|^{\alpha_{3}}\right),
\end{gathered}
$$

where $a \in\left\{a_{j l}, a_{j}, a_{0}\right\}$;

3) $\exists H_{4}>0 \forall\left\{(t, x),\left(t, \xi^{(i)}\right),\left(t, z^{(s)}\right) \mid i<s, i \in\{1,2\}, s \in\{2,3\}\right\} \subset \Pi_{[0, T]} \forall h \in[0, T]$ :

$$
\left|\Delta_{x_{i}}^{z_{i}} \Delta_{x_{s}}^{\xi_{s}} a_{j l}(t, x)\right| \leqslant H_{4}\left|x_{i}-z_{i}\right|^{\alpha_{i}}\left(h^{m_{s} \alpha_{s}}+\left|X_{s}(h)-\xi_{s}\right|^{\alpha_{s}}\right) ;
$$

4) there exist bounded and Hölder continuous in the sense (10)-(12) in $\Pi_{[0, T]}$ derivatives $\partial x_{1 j} \partial x_{1 l} a_{j l}$ and $\partial x_{1 j} a_{j}$.

The following theorem is valid.

Theorem 1. If the conditions 1-3 are satisfied, then there exists a classical FSCP $Z$ for the equation (5) with the estimates

$$
\begin{gathered}
\left|\partial_{x}^{k} Z(t, x ; \tau, \xi)\right| \leqslant C(t-\tau)^{-M-M_{k}} E_{c}(t, x ; \tau, \xi), \\
|S Z(t, x ; \tau, \xi)| \leqslant C(t-\tau)^{-M-1} E_{c}(t, x ; \tau, \xi),
\end{gathered}
$$

where $0 \leqslant \tau<t \leqslant T,\{x, \xi\} \subset \mathbb{R}^{n}, C$ and $c$ are positive constants, $m_{j}:=j-1 / 2, j \in\{1,2,3\}$, $M:=m_{1} n_{1}+m_{2} n_{2}+m_{3} n_{3}, M_{k}:=m_{1}\left|k_{1}\right|+m_{2}\left|k_{2}\right|+m_{3}\left|k_{3}\right|, k \in \mathbb{Z}_{+}^{n},\left|k_{j}\right|:=k_{j 1}+\ldots+k_{j n_{j}}$, $E_{c}(t, x ; \tau, \xi):=\exp \left\{-c \sum_{s=1}^{3}(t-\tau)^{1-2 s}\left|X_{s}(t-\tau)-\xi\right|^{2}\right\}, m_{1}\left|k_{1}\right|+\left|k_{2}\right|+\left|k_{3}\right| \leqslant 1$.

Proof. The proof of the theorem 1 uses the Levi method [22] and for more general case (complex-valued coefficients of equation (5)) is given in papers [23, 24].

If addition, the condition 4 is satisfied then a FSCP $Z$ has such properties: 
Property 1 (normality). Let $Z$ be a FSCP for the initial equation (5), and $Z^{*}$ be a FSCP for the adjoint equation (6). Then

$$
Z^{*}(\tau, \xi ; t, x)=Z(t, x ; \tau, \xi), \quad 0 \leqslant \tau<t \leqslant T, \quad\{\xi, x\} \subset \mathbb{R}^{n} .
$$

A FSCP $Z$ satisfying this equality is called a normal FSCP (NFSCP).

Proof. By virtue of the estimates (6) for $Z$ and similar estimates for $Z^{*}$, the formula (6) is valid. In this formula we set $u(\theta, y)=Z(\theta, y ; \tau, \xi), v(\theta, y)=Z^{*}(\theta, y ; t, x), t_{1}=\tau+\varepsilon$, and $t_{2}=t-\varepsilon$, where $\varepsilon$ is a sufficiently small positive number. Then we obtain the equality

$$
\int_{\mathbb{R}^{n}} Z^{*}(\tau+\varepsilon, y ; t, x) Z(\tau+\varepsilon, y ; \tau, \xi) d y=\int_{\mathbb{R}^{n}} Z^{*}(t-\varepsilon, y ; t, x) Z(t-\varepsilon, y ; \tau, \xi) d y,
$$

which, after passing to the limit, as $\varepsilon \rightarrow 0$, implies the required equality (15).

Property 2 (the convolution formula). The function $Z$ is a solution of the functional equation

$$
Z(t, x ; \tau, \xi)=\int_{\mathbb{R}^{n}} Z(t, x ; \lambda, y) Z(\lambda, y ; \tau, \xi) d y, \quad 0 \leqslant \tau<t \leqslant T, \quad\{x, \xi\} \subset \mathbb{R}^{n} .
$$

Proof. Just as in the proof of Property 1, we come to the equality

$$
\int_{\mathbb{R}^{n}} Z^{*}(\lambda, y ; t, x) Z(\lambda, y ; \tau, \xi) d y=\int_{\mathbb{R}^{n}} Z^{*}(t-\varepsilon, y ; t, x) Z(t-\varepsilon, y ; \tau, \xi) d y .
$$

The equality (17) is obtained when we pass to the limit in (18), as $\varepsilon \rightarrow 0$, and use the formula (15).

The equation (17) is called the Chapman-Kolmogorov equation. It expresses an important fact that a stochastic process is of the Markov type (a process without aftereffect). An investigation of the process can be based on the equation (17).

Property 3 (the uniqueness of the NFSCP). There exists only one NFSCP for the equation (5) satisfying the estimates (14).

Proof. Let $Z_{1}$ and $Z_{2}$ be two NFSCPs for the equation (5), both satisfying (14). We use the formula (8) setting $u(\theta, y)=Z_{1}(\theta, y ; \tau, \xi), v(\theta, y)=Z_{2}(t, x ; \theta, y)$. Then

$$
\int_{\mathbb{R}^{n}} Z_{1}\left(t_{2}, y ; \tau, \xi\right) Z_{2}\left(t, x ; t_{2}, y\right) d y=\int_{\mathbb{R}^{n}} Z_{1}\left(t_{1}, y ; \tau, \xi\right) Z_{2}\left(t, x ; t_{1}, y\right) d y .
$$

Since $t_{1}$ and $t_{2}$ from the interval $(\tau, t)$ are arbitrary, the last equality means that the function

$$
\int_{\mathbb{R}^{n}} Z_{1}(\theta, y ; \tau, \xi) Z_{2}(t, x ; \theta, y) d y, \quad \theta \in(\tau, t), \quad\{x, \xi\} \subset \mathbb{R}^{n},
$$

does not depend on $\theta$. Denote this function by $\Phi(t, x ; \tau, \xi)$. Thus,

$$
\Phi(t, x ; \tau, \xi)=\int_{\mathbb{R}^{n}} Z_{1}(\theta, y ; \tau, \xi) Z_{2}(t, x ; \theta, y) d y .
$$

Letting in the equality (19) first $\theta \rightarrow \tau$, and then $\theta \rightarrow t$, we find that

$$
\Phi(t, x ; \tau, \xi)=Z_{2}(t, x ; \tau, \xi)=Z_{1}(t, x ; \tau, \xi), \quad 0 \leqslant \tau<t \leqslant T, \quad\{x, \xi\} \subset \mathbb{R}^{n} .
$$

Our diffusion process is characterized by the diffusion matrix $A(t, x):=\left(a_{j l}(t, x)\right)_{j=1, l=1}^{n_{1}, n_{1}},(t, x) \in$ $\Pi_{(0, T]}$, and the drift vector $a(t, x):=\left(a_{1}(t, x), \ldots, a_{n_{1}}(t, x)\right),(t, x) \in \Pi_{(0, T]}$, whose elements are the appropriate coefficients of the equation (5). Let us express these important characteristics via the transition density of the stochastic process, that is the FSCP $Z$. 
Property 4 (a representation of the diffusion matrix and the drift vector via the function $Z$ ). The following formulas are valid:

$$
\begin{aligned}
& a(t, x)=\lim _{\tau \rightarrow t}\left((t-\tau)^{-1} \int_{\mathbb{R}^{n}}\left(y_{1}-x_{1}\right) Z(t, x ; \tau, y) d y\right) \\
& A(t, x)=\left(2^{-1} \lim _{\tau \rightarrow t}\left((t-\tau)^{-1} \int_{\mathbb{R}^{n}}\left(y_{1 j}-x_{1 j}\right)\left(y_{1 l}-x_{1 l}\right) Z(t, x ; \tau, y) d y\right)\right)_{j=1, l=1}^{n_{1}, n_{1}}, \quad(t, x) \in \Pi_{(0, T]} .
\end{aligned}
$$

Proof. It is sufficient to give the proof for the first coordinate $a_{1}$ of the vector $a$ and the element $a_{11}$ of the matrix $A$. Let us prove, for $(t, x) \in \Pi_{(0, T]}$, the formulas

$$
\begin{aligned}
& a_{1}(t, x)=\lim _{\tau \rightarrow t}\left((t-\tau)^{-1} \int_{\mathbb{R}^{n}}\left(y_{11}-x_{11}\right) Z(t, x ; \tau, y) d y\right), \\
& a_{11}(t, x)=2^{-1} \lim _{\tau \rightarrow t}\left((t-\tau)^{-1} \int_{\mathbb{R}^{n}}\left(y_{11}-x_{11}\right)^{2} Z(t, x ; \tau, y) d y\right) .
\end{aligned}
$$

Their proof is based on the formula (8), in which we set $u(\theta, y)=y_{11}-x_{11}$ and $v(\theta, y)=Z(t, x ; \theta, y)$. Then we obtain the equality

$$
-\int_{t_{1}}^{t_{2}} d \theta \int_{\mathbb{R}^{n}} Z(t, x ; \theta, y)\left(a_{1}(\theta, y)+a_{0}(\theta, y)\left(y_{11}-x_{11}\right)\right) d y=\left.\int_{\mathbb{R}^{n}}\left(y_{11}-x_{11}\right) Z(t, x ; \theta, y)\right|_{\theta=t_{1}} ^{t_{2}} d y
$$

Next in (22) we put $t_{1}=\tau, t_{2}=t-\varepsilon$, pass to the limit, as $\varepsilon \rightarrow 0$, divide the result by $t-\tau$ and obtain the equality

$$
\begin{array}{r}
(t-\tau)^{-1} \int_{\tau}^{t} d \theta \int_{\mathbb{R}^{n}} Z(t, x ; \theta, y) a_{1}(\theta, y) d y=(t-\tau)^{-1} \int_{\mathbb{R}^{n}}\left(y_{11}-x_{11}\right) Z(t, x ; \tau, y) d y \\
-(t-\tau)^{-1} \int_{\tau}^{t} d \theta \int_{\mathbb{R}^{n}} Z(t, x ; \theta, y) a_{0}(\theta, y)\left(y_{11}-x_{11}\right) d y .
\end{array}
$$

The equality (20) follows directly from (23), since the limit of the left-hand side, as $\tau \rightarrow t$, coincides with $a_{1}(t, x)$ due to properties of the FSCP $Z$ and the mean value theorem for integrals, while the second summand of the right-hand side of the equality (23) tends to zero by our assumptions regarding the function $a_{0}$. In order to prove (21), in the formula (8) we set $u(\theta, y)=\left(y_{11}-x_{11}\right)^{2}$ and $v(\theta, y)=$ $Z(t, x ; \theta, y)$. Then this formula can be re-written as

$$
\begin{aligned}
-\int_{t_{1}}^{t_{2}} d \theta \int_{\mathbb{R}^{n}} Z(t, x ; \theta, y)\left(2 a_{11}(\theta, y)+2 a_{1}(\theta, y)\left(y_{11}-x_{11}\right)+\right. & \left.a_{0}(\theta, y)\left(y_{11}-x_{11}\right)^{2}\right) d y \\
& =\left.\int_{\mathbb{R}^{n}}\left(y_{11}-x_{11}\right)^{2} Z(t, x ; \theta, y)\right|_{\theta=t_{1}} ^{t_{2}} d y .
\end{aligned}
$$

Then we repeat the reasoning from the previous case and come to the equality

$$
\begin{aligned}
(t-\tau)^{-1} \int_{\tau}^{t} d \theta & \int_{\mathbb{R}^{n}} Z(t, x ; \theta, y) a_{11}(\theta, y) d y=(2(t-\tau))^{-1} \int_{\mathbb{R}^{n}}\left(y_{11}-x_{11}\right)^{2} Z(t, x ; \tau, y) d y \\
& -(t-\tau)^{-1} \int_{\tau}^{t} d \theta \int_{\mathbb{R}^{n}}\left(a_{1}(\theta, y)\left(y_{11}-x_{11}\right)+2^{-1} a_{0}(\theta, y)\left(y_{11}-x_{11}\right)^{2}\right) Z(t, x ; \theta, y) d y
\end{aligned}
$$

which, after the above arguments, implies the formula (21). 
Similar reasoning leads to a representation via the function $Z$ for the function $-a_{0}$, the absorption coefficient of the diffusion process whose evolution is described by the Fokker-Planck-Kolmogorov equation (5).

Property 5 (a representation of the coefficient $a_{0}$ via the function $Z$ ). The following formula is valid:

$$
a_{0}(t, x)=\lim _{\tau \rightarrow t}\left((t-\tau)^{-1}\left(\int_{\tau}^{t} d \theta \int_{\mathbb{R}^{n}} Z(t, x ; \theta, y) d y-1\right)\right), \quad(t, x) \in \Pi_{(0, T]} .
$$

Property 6 (positivity of the function $Z$ ). The following inequality is valid

$$
Z(t, x ; \tau, \xi)>0, \quad 0 \leqslant \tau<t \leqslant T, \quad\{x, \xi\} \subset \mathbb{R}^{n} .
$$

Proof. Let $g$ be a continuous non-negative function with a compact support. Consider the function

$$
v(t, x):=\int_{\mathbb{R}^{n}} Z(t, x ; \tau, \xi) g(\xi) d \xi, \quad(t, x) \in \Pi_{(\tau, T]} .
$$

The function $v$ satisfies the conditions of [1, p.208, Theorem 3.16]. Indeed, it is a solution of the equation (5), that is $(L v)(t, x)=0,(t, x) \in \Pi_{(\tau, T]}$, satisfies the conditions $v(\tau, x)=g(x) \geqslant 0$, $x \in \mathbb{R}^{n}$, and $\lim _{|x| \rightarrow \infty} v(t, x) \geqslant 0, t \in(\tau, T]$. Therefore, by [1, p. 208, Theorem 3.16] we have $v(t, x) \geqslant 0$, $(t, x) \in \Pi_{(\tau, T]}$.

Now we take a delta-like sequence of functions $g_{\nu}, \nu \geqslant 1$, such that $g_{\nu}(\xi)=0$ for $\left|\xi-\xi^{0}\right|>1 / \nu$, and $\int_{\mathbb{R}^{n}} g_{\nu}(\xi) d \xi=1$, and denote by $v_{\nu}$ the function (24) corresponding to $g=g_{\nu}$ and $\tau=\tau^{0}$. Then for arbitrary fixed points $\left(t^{0}, x^{0}\right)$ and $\left(\tau^{0}, \xi^{0}\right)$, with $\tau^{0}<t^{0}$, we have $\lim _{\nu \rightarrow \infty} v_{\nu}\left(t^{0}, x^{0}\right)=Z\left(t^{0}, x^{0} ; \tau^{0}, \xi^{0}\right)$. Since $v_{\nu}\left(t^{0}, x^{0}\right) \geqslant 0$ for all $\nu \geqslant 1$, we find that $Z\left(t^{0}, x^{0} ; \tau^{0}, \xi^{0}\right) \geqslant 0$. Finally, observing that for every fixed point $(\tau, \xi)$ the function $Z(t, x ; \tau, \xi),(t, x) \in \Pi_{(\tau, T]}$, is not a constant identically, and using the strong maximum principle, we come to the strict positivity of the function $Z$.

Property 7 (a lower estimate of the function $Z$ ). There exists such a number $\Delta \in(0, T)$ that for any $t_{0} \in[0, T-\Delta],(t, x) \in \Pi_{\left(t_{0}, t_{0}+\Delta\right]}$, and $\delta \in\left(0, t-t_{0}\right)$ there exist such numbers $\omega>0$ and $\gamma>0$ that

$$
Z(t, x ; \tau, \xi) \geqslant \omega \exp \left\{-\gamma|\xi|^{2}\right\}, \quad(\tau, \xi) \in \Pi_{\left[t_{0}, t-\delta\right]} .
$$

Proof. The proof is similar to proof of the property 3.13 [1, p. 214].

\section{Correct solvability of the Cauchy problem}

The results from Section 2 and paper [25] allow us to investigate properties of the potentials generating by FSCP $Z$ and based on these properties prove different theorems about correct solvability of the Cauchy problem for the equation (5). Present some of them. The FSCP $Z$ generates generalized heat potential - the Poisson integral.

$$
u_{1}(t, x):=(P \varphi)(t, x):=\int_{\mathbb{R}^{n}} Z(t, x ; 0, \xi) \varphi(\xi) d \xi, \quad(t, x) \in \Pi_{(0, T]},
$$

of a function $\varphi$, the Poisson integral

$$
u_{0}(t, x):=(P \mu)(t, x):=\int_{\mathbb{R}^{n}} Z(t, x ; 0, \xi) d \mu(\xi), \quad(t, x) \in \Pi_{(0, T]},
$$

of a generalized measure $\mu$.

The exact estimates of the FSCP make it possible to prove precise theorems of the correct solvability of the Cauchy problem. The construction given below is a general framework for such theorems, and 
also for the theorems on the representation of solutions defined on an open layer $\Pi_{(0, T]}$, in terms of their limit values on the hyperplane $\{t=0\}$. The essence of the construction is a characterization of the evolution of a solution of the Cauchy problem in $t$ by their belonging to a family of Banach spaces (a specific space for each value of $t$ ).

Consider the equation (5) with the condition on the lower boundary of the layer $\Pi_{(0, T]}$ (the initial condition)

$$
\left.u\right|_{t=0}=\varphi .
$$

Denote by $\Phi$ such a space of initial data that for any $\Phi$ there exists a classical solution $u$ of the Cauchy problem (5), (27). Let $U$ be the space of all such solutions $u$.

$\Phi$ is the space of functions increasing exponentially as $|x| \rightarrow \infty$, then the appropriate space $U$ consists of functions which increase exponentially for $|x| \rightarrow \infty$ as functions of $x$, for every fixed $t \in(0, T]$. The type of growth may depend on $t$. That means that a solution $u(t, x),(t, x) \in \Pi_{(0, T]}$, as a function of $x$ for each fixed $t \in(0, T]$, belongs to a certain space $U_{t}$. Hence the temporal evolution of solutions of the problem (5), (27) can be described by their belonging to the appropriate spaces $U_{t}$.

We shall use necessary norms and spaces. Define the functions

$$
\begin{gathered}
\boldsymbol{k}(t, \boldsymbol{a}):=\left(k_{1}\left(t, a_{1}\right), k_{2}\left(t, a_{2}\right), k_{3}\left(t, a_{3}\right)\right) ; \quad \ell(t):=\left(\ell_{1}(t), \ell_{2}(t), \ell_{3}(t)\right) ; \\
k_{s}\left(t, a_{s}\right):=c_{0} a_{s}\left(c_{0}-a_{s} t^{2 s-1}\right)^{-1}, \quad s \in\{1,2,3\} ; \\
\ell_{1}(t):=k_{1}\left(t, a_{1}\right)+2 t^{2} k_{2}\left(t, a_{2}\right)+t^{4} k_{3}\left(t, a_{3}\right), \\
\ell_{2}(t):=2 k_{2}\left(t, a_{2}\right)+4 t^{2} k_{3}\left(t, a_{3}\right), \quad \ell_{3}(t):=4 k_{3}\left(t, a_{3}\right), \quad t \in[0, T],
\end{gathered}
$$

where $c_{0} \in(0, c), c$ is the constant from estimates $\operatorname{FSCP}(21), \boldsymbol{a}:=\left(a_{1}, a_{2}, a_{3}\right)$ are such set nonnegative numbers that $T \leqslant \min _{s \in\{1,2,3\}}\left(c_{0} / a_{s}\right)^{1 /(2 s-1)}$.

Let us $p \in[1, \infty]$ and $u(t, x),(t, x) \in \Pi_{[0, T]}$ be a given function, measurable in $x$ for any $t \in[0, T]$. For every $t \in[0, T]$ we define the norms

$$
\begin{gathered}
\|u(t, \cdot)\|_{p}^{\boldsymbol{k}(t, \boldsymbol{a})}:=\left\|u(t, x) \exp \left\{-\sum_{s=1}^{3} k_{s}\left(t, a_{s}\right)\left|X_{s}(t)\right|^{2}\right\}\right\|_{L_{p}\left(\mathbb{R}^{n}\right)}, \\
\|u(t, \cdot)\|_{p}^{\ell(t)}:=\left\|u(t, x) \exp \left\{-\sum_{s=1}^{3} \ell_{s}(t)\left|x_{s}\right|^{2}\right\}\right\|_{L_{p}\left(\mathbb{R}^{n}\right)} .
\end{gathered}
$$

We shall use the following spaces: $L_{p}^{\boldsymbol{k}(t, \boldsymbol{a})}, t \in[0, T], p \in[1, \infty]$, the spaces of measurable functions $\varphi: \mathbb{R}^{n} \rightarrow R$, with finite norms $\|\varphi\|_{p}^{\boldsymbol{k}(t, \boldsymbol{a})} ; L_{p}^{\boldsymbol{a}}:=L_{p}^{\boldsymbol{k}(0, \boldsymbol{a})} ; M^{\boldsymbol{a}}$ is the space of generalized Borel measures $\mu$ on $\mathbb{R}^{n}$ satisfying the condition

$$
\|\mu\|^{\boldsymbol{a}}:=\int_{\mathbb{R}^{n}} \exp \left\{-\sum_{s=1}^{3} a_{s}\left|x_{s}\right|^{2}\right\} d|\mu|(x)<\infty,
$$

where $|\mu|$ is the total variation of $\mu ; L_{1}^{-\ell(T)}$ is the space of measurable functions $\psi: \mathbb{R}^{n} \rightarrow \mathbb{R}$ with a finite norm

$$
\left\|\psi(x) \exp \left\{\sum_{s=1}^{3} \ell_{s}(T)\left|x_{s}\right|^{2}\right\}\right\|_{L_{1}\left(\mathbb{R}^{n}\right)} ;
$$

$C_{0}^{-\ell(T)}$ is the space of continuous functions $\psi: \mathbb{R}^{n} \rightarrow \mathbb{R}$, such that $|\psi(x)| \exp \left\{\sum_{s=1}^{3} \ell_{s}(T)\left|x_{s}\right|^{2}\right\} \rightarrow 0$, as $|x| \rightarrow \infty$. 
Theorem 2. Suppose that the conditions 1-4 are satisfied. Then:

1) for each function $\varphi \in L_{p}^{a}, p \in[1, \infty]$, the formula (25) determines the unique solution of the equation (5), for which

$$
\left\|u_{1}(t, \cdot)\right\|_{p}^{\boldsymbol{k}(t, \boldsymbol{a})} \leqslant C\|\varphi\|_{p}^{\boldsymbol{a}}, \quad t \in(0, T]
$$

and

$$
\lim _{t \rightarrow 0}\left\|u_{1}(t, \cdot)-\varphi(\cdot)\right\|_{p}^{\ell(t)}=0
$$

if $p \in[1, \infty)$, or

$$
\lim _{t \rightarrow 0} \int_{\mathbb{R}^{n}} \psi(x) u_{1}(t, x) d x=\int_{\mathbb{R}^{n}} \psi(x) \varphi(x) d x
$$

for any function $\psi \in L_{1}^{-\ell(T)}$ if $p=\infty$;

2) for each generalized measure $\mu \in M^{a}$, the formula (26) determines the unique solution of the equation (5), for which

$$
\left\|u_{0}(t, \cdot)\right\|_{1}^{\boldsymbol{k}(t, \boldsymbol{a})} \leqslant C\|\mu\|^{\boldsymbol{a}}, \quad t \in(0, T],
$$

and

$$
\lim _{t \rightarrow 0} \int_{\mathbb{R}^{n}} \psi(x) u_{1}(t, x) d x=\int_{\mathbb{R}^{n}} \psi(x) \varphi(x) d x
$$

and for any function $\psi \in C_{0}^{-\ell(T)}$.

Constant $C>0$ does not depend on a function $\varphi \in L_{p}^{a}$ and on a generalized measure $\mu \in M^{a}$ in $(28),(31)$.

The following theorem is inverse (in same sense) to the theorem 2.

Theorem 3. Suppose that the coefficients of equation (5) satisfy the conditions 1-4, and the solution $u$ is defined in the layer $\Pi_{(0, T]}$ and satisfies following conditions

$$
\|u(t, \cdot)\|_{p}^{\boldsymbol{k}(t, \boldsymbol{a})} \leqslant C, \quad t \in(0, T],
$$

with some constants $C>0$ and $p \in[1, \infty]$. Then, if $p \in(1, \infty]$, then exists a unique function $\varphi \in L_{p}^{a}$, and if $p=1$, then exists a unique generalized measure $\mu \in M^{a}$ such that the solution $u$ is represented by the formula (26) or (27).

From the theorem 2 and the theorem 3, under conditions 1-4 on the coefficients of the equation (5), follow such statements:

Corollary 1. The spaces $L_{p}^{a}$ and $M^{a}$ are the sets of initial values of the solutions for the equation (5) if and only if these solutions satisfy condition (33) with $p \in(1, \infty]$ and $p=1$, respectively.

Corollary 2. The solution $u$ of the equation (5) has representations in the form (26) or (27) with $\phi \in L_{p}^{a}$ and $\mu \in M^{\boldsymbol{a}}$ if and only if condition (33) is valid.

Corollary 3. Under the condition (33), solutions of the equation (5) satisfy initial condition with $t=0$ in the sense of (29), (30) and (32), respectively.

In the end, formulate one theorem the proof of which is based on maximum principal for solutions of the equation (5).

Theorem 4. Suppose that conditions 1-4 are satisfied. Then the Cauchy problem for the equation (5), cannot have more then one nonnegative solution.

[1] Eidelman S. D., Ivasyshen S. D., Kochubei A. N. Analytic Methods in the Theory of Differential and PseudoDifferential Equations of Parabolic Type. Birkhäuser Verlag, Basel-Boston-Berlin (2004). 
[2] Kolmogoroff A. N. Über die analytischen Methoden in der Wahrscheinlichkeitsrechnung. Mathematische Annalen. 104, 415-458 (1931).

[3] Einstein A. Investigations on the Theory of the Brownian Movement. Dover, New-York (1956).

[4] Einstein A., von Smoluchowski M. Brownsche Bewegung. Verlag Harri Dentsch, Frankfurt am Main (1997).

[5] Uhlenbeck E. B., Ornstein L. S. On the theory of Brownian motion. Phys. Rev. 36, 823-841 (1930).

[6] Kolmogoroff A. N. Zufällige Bewegungen (zur Theorie der Brownschen Bewegung). Annals of Mathematics. Second Series. 35 (1), 116-117 (1934).

[7] Dynkin E. B. Markov Processes I, II. Springer, Berlin (1965).

[8] Doob I. L. Stochastic Processes. Wiley, New York (1953).

[9] Portenko N. I. Generalized Diffusion Processes. Vol. 83. Am. Math. Soc. (1990).

[10] Sonin I. M. On a class of degenerate diffusion processes. Theor. Probab. Appl. 12 (3), 49-496 (1967).

[11] Ivasyshen S. D., Medynsky I. P. The Fokker-Planck-Kolmogorov equations for some degenerate diffusion processes. Theory of Stochastic Processes. 16(32) (1), 37-66 (2010).

[12] Citti G., Pascucci A., PolidoroS. On the regularity of solutions to a nonlinear ultraparabolic equations arising in mathematical finance. Differ. Integral Equat. 14 (6), 701-739 (2001).

[13] Pascucci A., Polidoro S., Lanconelli E. Linear and nonlinear ultraparabolic equations of Kolmogorov type arising in diffusion theory and in finance. Nonlinear Problems in Mathematical Physics and Related Topics, vol. II. In Honor of Professor O. A. Ladyzhenskaya. International Mathematical Series, pp. 243-265 (2002).

[14] Pascucci A. Kolmogorov Equations in Physics and in Finance. In: Bandle C. et al. (eds) Elliptic and Parabolic Problems. Progress in Nonlinear Differential Equations and Their Applications, vol. 63, pp. 353364 (2005).

[15] Di Francyesco M., Pascucci A. A cotinuous dependence result for ultraparabolic equations in option pricing. J. Math. Anal. Appl. 336 (2), 1026-1041 (2007).

[16] Di Francyesco M., Pascucci A. On a class of degenerate parabolic equations of Kolmogorov type. Applied Mathematics Research eXpress. 2005 (3), 77-116 (2005).

[17] Foschi P., Pascucci A. Kolmogorov equationsa arising in finance: direct and inverce problems. Lect. Notes of Seminario Interdisciplinare di Matematica. Universita degli Studi della Basilicata. VI, 145-156 (2007).

[18] Lanconelli E., Polidoro S. On a class of hypoelliptic evolution operators. Rend. Sem. Mat. Univ. Politec. Torino. Partial Diff. Eqs. 52 (1), 29-63 (1994).

[19] Polidoro S. On a class of ultraparabolic operators of Kolmogorov-Fokker-Planck type. Le Matematiche. 49 (1), 53-105 (1994).

[20] Bogachev N. U., Krylov N.V., Röckner M., Shaposhnikov S.V. Fokker-Planck-Kolmogorov equations. Vol. 207. Am. Math. Soc. (2015).

[21] Bogachev N. U., Röckner M., Shaposhnikov S. V. On convergence to stationary distributions for solutions of nonlinear Fokker-Planck-Kolmogorov equations. J. Math. Sci. 242 (1), 69-84 (2019).

[22] Ivasyshen S. D., Medynsky I. P. On aplications of the Levi method in the theory of parabolic equations. Matematychni Studii. 47 (1), 33-46 (2017).

[23] Ivasyshen S. D., Medynsky I. P. The classical fundamental solution of the Cauchy problem for ultraparabolic Kolmogorov type equations with two groups spatial variables of degeneration. I. Mat. Metody Fiz.-Mech. Polya. 60 (3), 9-31 (2017), (in Ukrainian).

[24] Ivasyshen S. D., Medynsky I. P. The classical fundamental solution of the Cauchy problem for ultraparabolic Kolmogorov type equations with two groups spatial variables of degeneration. II. Mat. Metody Fiz.-Mech. Polya. 60 (4), 7-24 (2017), (in Ukrainian).

[25] Ivasyshen S. D., Medyns'kyi I. P. On the Classical Fundamental Solutions of the Cauchy Problem for Ultraparabolic Kolmogorov-Type Equations with Two Groups of Spatial Variables. J. Math. Sci. 231, 507-526 (2018). 


\title{
Про властивості розв'язків рівнянь Фоккера-Планка-Колмогорова
}

\author{
Мединський І. П. \\ Національний університет "Лъвівсъка політехніка", \\ вул. С. Бандери, 12, 79013, Львів, Україна
}

У статті висвітлюється зв'язок між дифузійними процесами і диференціальними рівняннями з частинними похідними параболічного типу. Зроблено акцент на вироджених параболічних рівняннях з дійсними коефіцієнтами. Ці рівняння є узагальненням класичного рівняння дифузії з інерцією Колмогорова. Такі рівняння природно розглядати як рівняння Фоккера-Планка-Колмогорова для відповідних вироджених дифузійних процесів. Фундаментальний розв'язок задачі Коші для рівняння Фоккера-Планка-Колмогорова визначає густину перехідних імовірностей відповідного дифузійного процесу. Сформульовано умови на коефіцієнти рівняння за яких існує класичний фундаментальний розв'язок задачі Коші і доведено ряд його основних властивостей, а також наведено застосування фундаментального розв'язку до дослідження коректної розв'язності задачі Коші.

Ключові слова: дифузійний прочес, перехідна ймовірність прочесу, рівняння Фоккера-Планка-Колмогорова, вироджене параболічне рівняння, фундаментальний розв'язок, задача Kоші. 